\title{
Bridging the Digital Divide for deaf signer users
}

\author{
Inmaculada Fajardo \\ University of Manchester \\ MBS, Booth Street east \\ M15 6PB, UK \\ Manchester, UK \\ ifajardo@manchester.ac.uk
}

\author{
Julio Abascal \\ University of the Basque Country \\ School of Informatics \\ Manuel Lardizabal 1 \\ San Sebastián, Spain \\ Julio.abascal@si.ehu.es
}

\author{
José Juan Cañas \\ University of Granada \\ Facultad de Psicología \\ Campus Cartuja sn \\ Granada, Spain \\ delagado@ugr.es
}

\begin{abstract}
Motivation - In order to design strategies to overcome deaf signer users' digital divide, the authors portray an overview of some of the cognitive processing characteristics of this type of users during Web information search based on insights gained from the Cogniweb project's empirical research.
\end{abstract}

Research approach - Critical review of 5 experiments on Web Information Search which manipulated system and users' variables.

Findings/Design - The results highlight the relevance of 3 cognitive factors as predictors of Web information search efficiency for deaf people: reading skills, verbal categorization abilities and visual-spatial span. Additionally, the results suggest that the usage of textual hyperlinks linked to videos with their Sing Language translations seems to be an efficient Web navigation mechanism for deaf signer users.

Research limitations/Implications - A small number of deaf signer users participated in the set of studies, which limited a possible generalisation of the findings.

Originality/Value - The research makes a contribution to the knowledge about how cognitive and systems factors interact and how this knowledge can be used to increase Web information scents for deaf signer people.

Take away message - Need of researchers to investigate Sign Language navigation mechanisms as an alternative to overcome the digital divide for users of linguistic minorities like deaf signers.

\section{Keywords}

Web information scent, cognitive processes, deafness, linguistic minority, sing language, digital divide

\section{INTRODUCTION}

A basic Internet literacy such as information search can be greatly compromised for linguistic minorities generally underrepresented in the net. That is particularly the case of the linguistic minority conformed by deaf people users of the Sing Language (SL). In this article, we gather and discuss the main insights gained from the project Cogniweb (IMSERSO,
2002-2007) aimed to study strategies to bridge the digital divide for deaf signer users.

In particular, Cogniweb approaches two objectives. On the one hand, it is intended to investigate the influence of diverse cognitive factors, which operate in a particular way in deaf people, in the information search task. These factors range from reading comprehension or visual span to verbal categorization abilities. On the other hand, the project examines how design factors such as hyperlink format (text, icons and videos in SL) and menu structure may interact with the mentioned cognitive factors to facilitate or interfere the information search process.

READING, VERBAL CATEGORIZATION AND VISUAL COGNITION IN DEAF PEOPLE

As found in a diversity of linguistic contexts, deaf population seems to present low levels of reading literacy (e.g. Asensio, 1989, Alegría 1999, GoldinMeadow \& Rachel 2001, Leybaert, Alegría \& Morais 1982). This is not a trivial fact that may affect, for instance, their rate of access to university studies. Villalba, Ferrer and Asensi (1999) observed that deaf student with low reading skill failed exams to access University studies in Spain while those deaf students who had a similar level of reading skill than hearing students passed them.

Likewise, as Luckner et al. highlight literacy skills are essential to success in today's technological society (Luckner, Sebald, Cooney, Young, \& Muir, 2005/2006, pp. 443). In particular, specific competencies like Web information search may be interfered by the lack of reading skills. During information search, users make navigation decisions by making semantic comparisons between their objectives and the set of choices, generally textual hyperlinks, displayed in a Web page (e.g. Card, Pirolli, Van Der Wege, Morrison, Schraedley $\&$ Boshart, 2001). If reading proficiency is low, this process can be interfered.

This hypothesis was actually explored in one of the experiments of Cogniweb where a group of 27 deaf signers were asked to search for specific news headlines in a digital newspaper and fulfil a reading test. The results showed that deaf participants' reading skills correlated significantly with percentage of targets found 
and with knowledge acquisition during the search task (Fajardo, Cañas, Salmerón \& Abascal, 2008). As expected, the higher the level of reading skill, the more targets found and the higher the knowledge acquisition about the Web structure.

The late acquisition of oral language and reading literacy by deaf people may impact as well the organization and access to the knowledge stored in the Long Term Memory. Some studies have found that deaf people under-perform their hearing peers in tasks which imply the use of taxonomical or categorical verbal information (e.g. Marschark, Convertino, McEvoy, \& Masteller, 2004; Marschark \& Everhart, 1999). A characteristic of some websites or hypertexts systems is the organization of categories of contents in a hierarchy of Web pages. For instance, most commercial Websites organize their good in categories so that users have to use their categorical knowledge to make navigation decisions. Therefore, deficiencies in verbal categorical reasoning could be partially responsible for the information search problems of deaf users.

For the preliminary exploration of this hypothesis, we carried out a study in collaboration with the University of Padova (Fajardo, Arfé, Benedetti \& Altoé, 2007). A group of 30 deaf signer and 31 hearing non signer students were asked to perform 2 tasks: a categorization test (to categorize items -exemplars- as belonging to one of two categories, Snodgrass and McCullogh, 1986) and Web information search (to find goods in a digital supermarket). Against our prediction, although deaf users were slower than hearing users in both the categorization test and the search task, categorization scores did not correlate with search efficiency, that is, categorization and Web search did not seem to be related.

In following experiments, we tested again this hypothesis using a more complex categorization test (Parra, 2007; Fajardo, Parra, Cañas et al., 2008). In particular, we used a Verbal Analogy Task in which users were asked to solve analogies like "banana is to fruit as rose is to ___ (flower)" by providing category or exemplar names. The rationale behind it was that deaf and hearing students could not differ as much in knowledge organization as in the automatic application of it (Marschark et al, 2004). The verbal analogy test does not demand explicitly the use of categorical knowledge as neither would do information search in hierarchical reasoning. Therefore, if deaf signers present difficulties in solving verbal analogies, they would predict difficulties in information search.

In fact, we observed that the efficiency in the application of verbal categories was lower for deaf than for hearing participants. Supporting our hypothesis, those deaf users who were more accurate using superordinate and sub-ordinate categories, were as well more efficient in a search task performed in a commercial website with textual hyperlinks organized hierarchically. Our conclusion was that in the late 2 experiments deaf users were not able to automatically activate a knowledge they actually had to solve the task. Then, if the verbal format is not appropriate for deaf signers, how can their categorical knowledge necessary to navigate in hierarchical websites be activated?

In the next sections we will describe how different information format (icons, sign language) seem to help in this purpose but before that we will finish this section mentioning other cognitive factor, visuo-spatial span, relevant of deaf user Web accessibility.

Despite the series of disadvantages highlighted in the previous paragraph regarding oral and printed language, deaf people can efficiently communicate by means of the SL. The SL is a natural language with its own grammatical structure which uses mainly hand and mouth movements to communicate meanings. This visuo-spatial nature of the SL could be improving the visuo-spatial memory of deaf signers not only for linguistic material (sings) but also for non-linguistic ones (Arnolds \& Mills, 2001). If menu and Website information search demand visual-spatial memory like some studies seem to suggest (Snowberry, Parkinson \& Sisson, 1983), deaf people could be using a visuospatial strategy to compensate their lack of reading proficiency.

This prediction was tested in one of the Cogniweb's experiments already cited (Fajardo, Arfé, Benedetti \& Altoé, 2007). The group of deaf students was asked to fulfill verbal and visuo-spatial span tests. It was found that while verbal span did not correlate with search, visuo-spatial span's performance did. Deaf students with higher spatial span were faster in graphical Web search. This result suggests that deaf users apply a visual-matching strategy more often than a semantic approach to make navigation decisions. Actually, Namatame and Kitajima's (2005) found that deaf participants used less semantic scan patterns (measure extracted from eye movement logs) of Webpage content comparing to hearing participants. Put together, both findings could support the hypothesis of deaf and hearing users using different strategies for Web information search.

In this section, we have shown how the Cogniweb's empirical series brings awareness about some cognitive factors which are critical for deaf users' information search. In the next sections, we describe the results related to the interaction between those cognitive factors and design factors.

\section{NARROW AND DEEP MENUS FOR OCCASIONAL WEBSITES}

A consistent finding in the literature about Web information structure is the effect of superiority of wide structures (many hyperlink choices per node or pages) over deep structures (hyperlinks distributed in many layers of nodes) for hearing users (e.g. Norman 1991; Zaphiris, Shneiderman \& Norman 2002). It is argued that deep structures may overload users' working memory due to the greater relational processing demand (Lee \& Tedder, 2004) required to guess distal items of 
information from proximal cues or choices displayed in the more superficial layer of nodes.

In Fajardo, Cañas, Salmerón and Abascal (2008) we tried to test the effect of superiority of wide structures for deaf users. Twenty-seven prelingual deaf people users of SL were asked to search a set of headlines in a Web newspaper where information structure and practice were manipulated. While practice did not affect deep structures (Web content distributed through 4 layers of nodes, see Figure 1), wide structures (Web content concentrated in 2 layers, see Figure 2) did facilitate users' performance in the last trial block and compromised it in the first trial block. It was argued that wide structures generated verbal information overload for deaf users, which decreased with practice. Thus, although in need of further research, we can conclude from these results that in the case of deaf signer users wide structures would be preferable for websites requiring frequent use, rather than for those intended for occasional interaction.

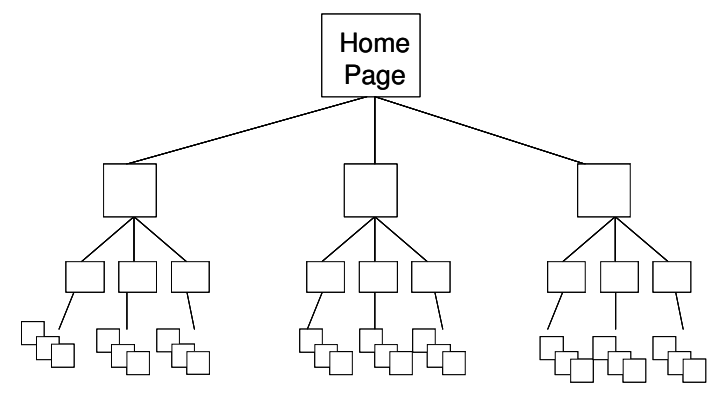

Figure 1. Sketch of the Deep Web structure $(8 \times 3 \times 3 \times 3)$ used in Fajardo et al. (2008).

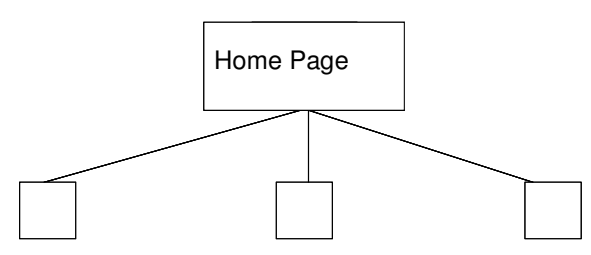

Figure 2. Sketch of the Wide Web structure $(62 \times 3)$ used in Fajardo et al. (2008).

Although the information structure may help, the major problem of deaf people is still conformed by the presence of textual contents. Therefore, another of the challenges of Cogniweb described in the next sections is to find alternatives to information search with text cues.

HYPERLINKS I: TEXTUAL VS. GRAPHICAL

As we said before, according to Web information search models, the judgment of semantic similarity between target and choices is an important process during Web information search (Scent-based Navigation and
Information Foraging in the ACT architecture-SNIFATC of Pirolli \& Fu, 2003 or Comprehension-based Linked model of Deliberate Search-CoLiDeS of Kitajima, Blackmon \& Polson, 2000).

The choices in a Website can be either textual hyperlinks, graphical hyperlinks (e.g. icons) or even videos. As textual hyperlinks appear to be one of the Web elements generating accessibility problems for deaf users, could graphical hyperlinks have a facilitative effect instead? In agreement with a classical effect in cognitive psychology, pictures would be superior to words in semantic tasks (e.g. Nelson, Reed, \& Walling, 1976; Paivio, 1991). Therefore, it would be possible to improve not only deaf students' but also hearing students' information search performance by using graphical hyperlinks instead of textual hyperlinks.

This hypothesis was tested in other of the Cogniweb's experiments (Fajardo, Cañas, Salmerón \& Abascal, 2006). In this study, deaf signer and hearing users were asked to find newspaper sections (e.g. Football section) in a digital paper. Hyperlink format (picture vs. word) was manipulated between groups, so that half of the users performed the task with verbal hyperlinks (see Figure 3) and the other half did the task with graphical hyperlinks (see figure 4). In addition, path length (number of layers of nodes that users needed to visit to find the target) was manipulated within subject (short-3 vs. medium-4 vs. long-5).

It was found that Hearing students were always better with verbal hyperlinks than with graphical ones. Deaf users found more targets using Graphical than Textual hyperlinks for targets located in shallow nodes of the hypertext structure (higher levels of the hypertext contents hierarchy). However, when the targets were located in deep layers of the hypertext (lower levels of the hypertext contents hierarchy), that is, when it is hypothesized that semantic processing becomes more difficult (Norman, 1991), there were no differences in accuracy between conditions. In this last condition, contrarily to our predictions, deaf users using textual hyperlinks were faster and became less disoriented than those using graphical hyperlinks.

The absence of graphical facilitation for targets placed in deep layers could be due to uncontrolled factors such as the visual distinctiveness, familiarity or concreteness of the concepts used. For instance, abstract concepts (e.g. "culture") would be processed faster by the verbal code than by the visual one (Paivio, 1986).

Therefore, in one of the previously cited experiments (Fajardo, Arfé, Benedetti \& Altoé, 2007), these results were replicated but using as hyperlinks' source a set of high typicality, frequency and familiarity icons and words (obtained from the Dell' Acqua, Lotto and Job 's database, 2000). In this occasion, only the condition of deep-targets was used. Results showed that deaf students were faster with graphical than with textual hyperlinks. These results mean that the strategy of using graphical hyperlinks to improve deaf users' Web 
information scent in deep structures seems to be only sensible with pictures representing high familiar and frequent concepts.

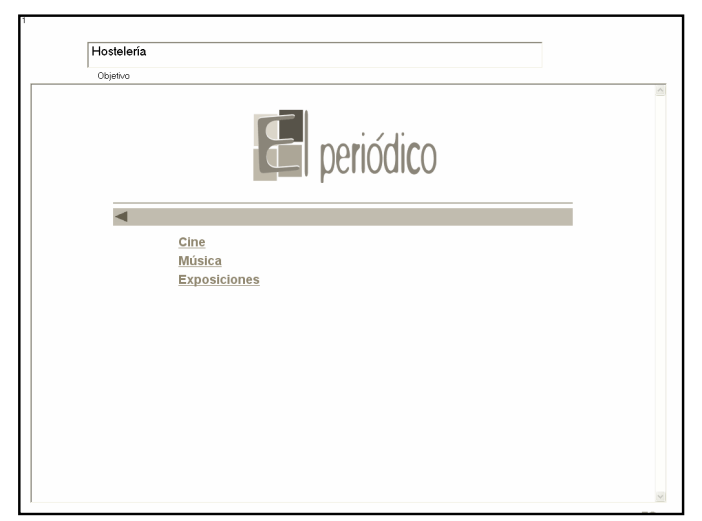

Figure 3. Node of the digital newspaper with verbal hyperlinks used in Fajardo et al. (2006).

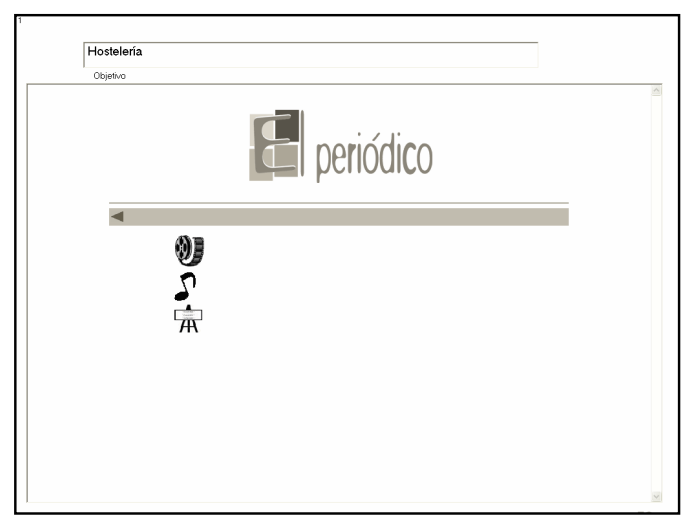

Figure 3. Node of the digital newspaper with graphical hyperlinks used in Fajardo et al. (2006).

Finally, as we mentioned before, thanks to the technological advances, hyperlinks could be also represented by videos with contents in SL. Theoretically, to allow deaf signer users to search for information in their native language would be the optimum alternative to bridge the digital divide. In the next section we describe two studies of Cogniweb conducted in which the efficiency of videos in SL to increase Web information scent was tested.

HYPERLINKS II: TEXTUAL SUPPORTED WITH SL VIDEOS

The inclusion of the SL in the Web it is not only an ethical goal which pretends to soft the disadvantages of deaf signers regarding information access but also a technological challenge. The technological advances have made possible that videos can be seen with high temporal and visual resolution in the Web. Even videos can now be encrypted in small frames of the websites preventing the necessity of opening a different window to play them and, therefore, reducing potential users' disorientation.

However, how would videos in SL affect the process of information search for deaf signers? It seems to be assumed that they will automatically increase the efficiency of the process, supposition that has not been empirically tested with deaf signer users though.

The last studies of the experimental series of Cogniweb have tried to explore the usability of two navigation mechanisms based on SL videos. In both mechanisms, the videos support textual hyperlinks by providing their corresponding translation to the SL performed by a SL expert. In version 1 (Figure 5), the videos were displayed always in the same frame located in the leftbottom corner of the screen. In version 2 , there were a small video frame per verbal hyperlink presenting a greater spatial contiguity video-textual hyperlink (Figure 6 and 7) placed in one of the mechanisms, In addition, the videos were clickable, that is, they were hyperlinks themselves.

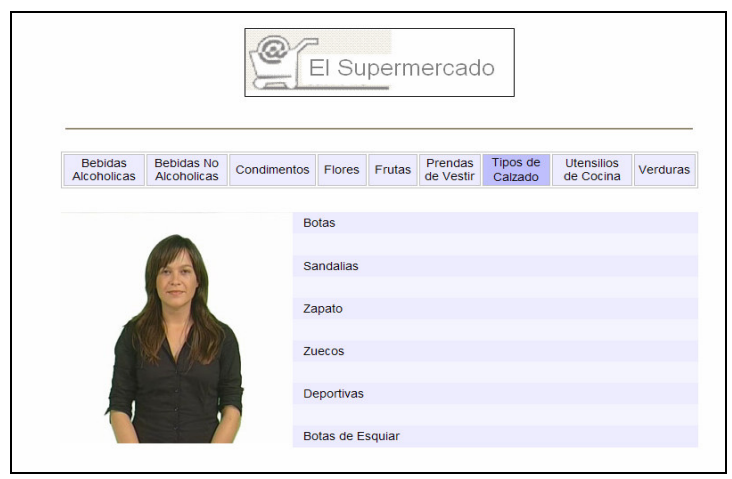

Figure 5. Node of the Digital supermarket with SL plus text hyperlinks (Version 1) used in Parra (2007).

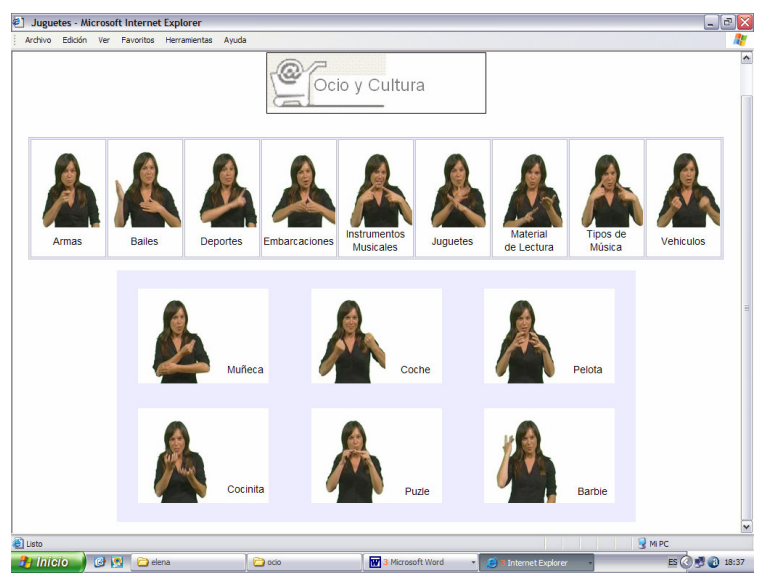

Figure 6. Node of the Digital supermarket with SL plus text hyperlinks (Version 2) used in Parra (2007). 


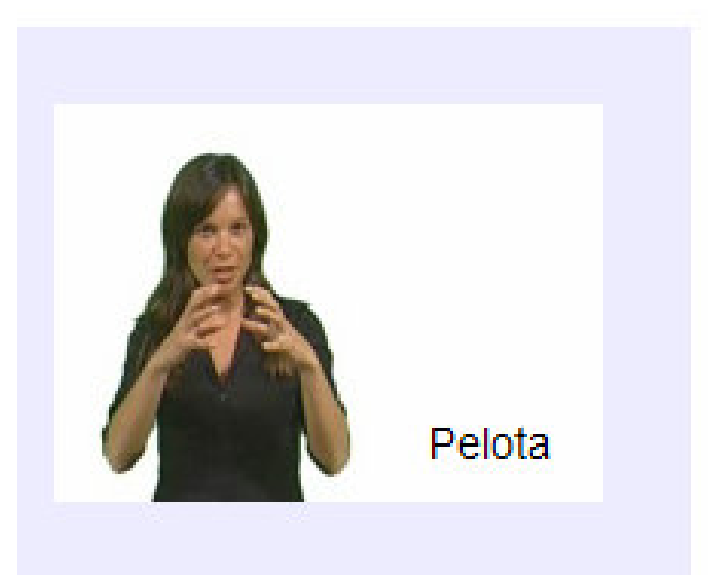

Figure 7. Detail of a SL plus text hyperlink used in Version 2 of Parra (2007).

In the first experiment (Parra 2007), 19 deaf signer users were asked to find 18 goods in each of the two versions of an on-line shop. The results showed that deaf users were more efficient with the clickable-contiguous version.

In the second experiment, this SL navigation mechanism was compared with a text-only version. This time, 23 deaf signer users were asked to perform a search task in both Website versions. Deaf signers became less disorientated (measured by the number of page visited per search) and were less dependent of their verbal categorical reasoning abilities using textual hyperlinks supported with videos in SL than just using textual hyperlinks. These results represent an empirical support to the usage of videos in SL linked to textual hyperlinks as an efficient Web navigation mechanism for deaf signer users.

\section{CONCLUSIONS}

This paper was intended to describe the main empirical findings of Cogniweb sketching some strategies to bridge the digital divide for deaf signer users.

Focusing on Web information search, we have brought awareness about some cognitive factors which have been found relevant to explain the problems that deaf users seem to find when following information scent cues in the Web. Reading literacy and automatic use of verbal categorical reasoning have been found to correlate negatively with information search time and disorientation of deaf signer users in hierarchically organized websites. Our findings also suggest that deaf users could be using a visual-matching strategy instead of following textual/semantic cues to search for information in a Website.

Regarding design factors, our results would imply some recommendations that, nevertheless, should be further tested before being implemented. Among them, we would recommend the usage of deep structures for websites of occasional use, graphical hyperlinks for very frequent and familiar concepts and the support of textual hyperlinks with videos in SL. Our research highlight as well the necessity of conducting further research to study the efficiency of videos in SL and other options to introduce SL in the Web such as Signer Avatars (Efthimiou, Fotinea \& Glauert, 2008) as the really unique alternative to overcome the digital divide for users of linguistic minorities like deaf signers.

\section{REFERENCES}

Alegría, J. (1999), La lectura en el niño sordo: elementos para una discusión [Reading in the deaf child: elements for discussion]. In: VV. AA. Actes du colloque: Lenguaje Escrito y Sordera. teóricos y derivaciones practicas, (Universidad de Salamanca: Publicaciones Universidad Pontificia de Salamanca), pp.59-76.

Arnolds, P., Mills, M., 2001. Memory for faces, shoes and objects by Deaf and Hearing Signers and Hearing Non-signers. Journal of Psycholinguistic research. 30 (2), 185-195.

Asensio, M. (1989). Los procesos de lectura en los deficientes auditivos. Tesis doctoral, UAM. In AA.VV. (1990). Resúmenes de Premios Nacionales de Investigación e Innovación Educativa. Madrid: CIDE, pp. 205-246.

Card, S., Pirolli, P., Van Der Wege, M., Morrison, Reeder, J., Schraedley, K. and Boshart, J (2001). Information scent as a driver of Web behavior graphs: results of a protocol analysis method for Web usability. In M. Beaudouin-Lafon, \& R.J.K. Jacob (Eds.), Proceedings of ACM CHI 2001 Human Factors in Computing Systems Conference (pp. 498-505). New York, NY: ACM Press.

Efthimiou, E., Fotinea, E. and Glauert, J. (2008). Special issue: "Emerging Technologies for Deaf Accessibility in the Information Society", Universal Access Information Society, 6:321-322.

Fajardo, I., Arfé, B, Benedetti, P. and Altoé, G.M. (2007). Hyperlink Format, Categorization Abilities and Memory Span as Contributors to Deaf Users Hypertext Access, Journal of Deaf Studies and Deaf Education, Advance Access published November 27, 2007, doi:10.1093/deafed/enm058.

Fajardo, I., Cañas, J.J., Salmerón, L. \& Abascal, J. (2006). Improving deaf users' accessibility in hypertext information retrieval: Are graphical interfaces useful for them? Behaviour \& Information Technology, 25(6): 455 - 467.

Fajardo, I., Parra, E., Cañas, J.J., Abascal, J., López J.M. y Gea, M. (2008). Web Textual Hyperlinks supported with Sign Language Videos, communication presented in the Workshop on Cognition and the Web 2008, Granada. (Spain), 26, April, 2008.

Goldin-Meadow, S. and Mayberry, R.I. (2001) How Do Profoundly Deaf Children Learn to Read?, Learning Disabilities Research \& Practice, 16, 222-229 
IMSERSO (2002-2006) COGNIWEB-IV. Estudio empírico de patrones de acceso a la web para mejorar la accesibilidad cognitiva de usuarios sordos. Financiado por: IMSERSO/Ministerio de Salud. Plan Nacional de Investigación Científica, Desarrollo e Innovación Tecnológica.

Kitajima, M., Blackmon, M.H., \& Polson, P.G. (2000). A comprehension based Model of Web Navigation and its Application to Web Usability Analysis. People and Computers XIV, 357-373.

Lee, M. J., and Tedder, M. C. (2004) Introducing expanding hypertext based on working memory capacity and the feeling of disorientation: Tailored communication through effective hypertext design. Journal of Educational Computing Research, 30, 171-195.

Leybaert, J., Alegria, J., and Morais, J. (1982). On automatic reading processes in the deaf. Cahiers de Psychologie Cognitive, 2, 185-192.

Luckner, J.L., Sebald, A.M., Cooney, J., Young, J., and Muir, S.G. (2005/2006). An examination of the evidence-based literacy research in deaf education. American Annals of the Deaf, 150(5), 443 - 456.

Marschark M., Convertino, C., McEvoy, C., and Masteller A. (2004) Organization and use of the mental lexicon by deaf and hearing individuals. American Annals of the Deaf, 149, 51-61.

Marschark, M., and Everhart, V.S. (1999). Problem solving by deaf and hearing children: Twenty Questions. Deafness and Education International, 1, 63-79.

Namatame, M., and Kitajima, M. (2005). Differences in Web interaction styles of hard-of-hearing and hearing persons. Paper presented at the $H C I$ International Conference, Las Vegas, Nevada.

Nelson, D.L., Reed, V.S. and Walling, J.R. (1976): Pictorical superiority effect. Journal of
Experimental Psychology; Human Learning and Memory, 5, 24-44.

Norman, K. (1991). The psychology of menu selection: Designing cognitive control of the human/computer interface. Norwood, NJ: Ablex.

Paivio, A. (1991) Dual-coding theory: Retrospect and current status. Canadian Journal of Psychology, 45: $255-287$.

Paivio, A. (1986). Mental representations. New York: Oxford University Press.

Parra, E. (2007) Influencia del uso de la Lengua de signos y la Organización del conocimiento categórico en la búsqueda de información Web por personas sordas, Universidad de Granada. Unpublished Master Thesis.

Pirolli, P., and Fu, W-T.F. (2003). SNIF-ACT :a model of information foraging on the World Wide Web. In proceeding of the Ninth International Conference on User Modeling.

Snodgrass, J.G., and McCullough, B. (1986). The role of visual similarity in picture categorization. Journal of Experimental Psychology: Learning, Memory and Cognition, 12, 147-154.

Snowbetry, K., Parkinson, S. R., and Sisson N. (1983). Computer display menus. Ergonomics, 26,7,699712.

Villalba, A., Ferrer, A., and Asensi, C. (1999). La comprensión lectora en universitarios con déficit auditivo. Logopedia, Foniatría y Audiología, XIX (1), 33-39.

Zaphiris, P., Shneiderman, B., and Norman, K.L. (2002) Expandable Indexes Versus Sequential Menus for Searching Hierarchies on the World Wide Web. Behaviour and Information Technology, 21 (3), 185-201 